\title{
Unsupervised Cluster Matching for Content Model
}

\author{
E. Suchitha \\ Department of CSE \\ SNIST, JNTUH, Telangana, India \\ Telangana, India
}

\author{
N. Venkata Subba Reddy \\ Department of CSE \\ SNIST, JNTUH, Telangana, India
}

\author{
Prasanta Kumar Sahoo, PhD \\ Department of CSE \\ SNIST, JNTUH
}

\begin{abstract}
People are generating huge amount of data which user need to store data in the storage devices. But storing the data in cloud is unsecure and people are storing the same data again and again. to avoid waste of storing the data again and again on same documents, we are going to use clustering the data documents for same documents and transform them into single language and store them. Whenever user need the document then it translate into user defined language and shows results to user. The application of document clustering can be categorized to two types, online and offline. Online applications are usually constrained by efficiency problems when compared to offline applications. Text clustering may be used for different tasks, such as grouping similar documents (news, tweets, etc.) and the analysis of customer/employee feedback, discovering meaningful implicit subjects across all documents. Documents can be clustered based on structure or content based meaning of the documents. In the existing system when documents are translated into user defined language documents are getting different meaning and it can convert into only two languages only.
\end{abstract}

\section{Keywords}

Data Mining, RSA, HSIC(HILBERT-SCHMIDT INDEPENDENCE CRITERION).

\section{INTRODUCTION}

Translation is the communication of the meaning of a source language text by means of an equivalent target language text The English language draws a terminology distinction (which does not exist in every language) between translating (a written text) and interpreting (oral or signed communication between users of different languages); under this distinction, translation can begin only after the appearance of writing within a language community. Consensus clustering is an important elaboration of traditional cluster analysis Consensus clustering, also called cluster ensembles or aggregation of clustering (or partitions), refers to the situation in which a number of different (input) clustering have been obtained for a particular dataset and it is desired to find a single (consensus) clustering which is a better fit in some sense than the existing clustering. By and large, interpreters have looked to protect the setting itself by replicating the first request of sememes, and thus word request-when essential, reconsidering the real syntactic structure, for instance, by moving from dynamic to aloof voice, or the other way around.

The syntactic contrasts between "fixed-word-request" dialects (for example English, French, German) and "free-wordrequest" languages(e.g., Greek, Latin, Polish, Russian) have been no obstacle right now. The specific linguistic structure (sentence-structure) attributes of a book's source language are acclimated to the syntactic prerequisites of the objective language. When a target language has lacked terms that are found in a source language, translators have borrowed those terms, thereby enriching the target language. Thanks in great measure to the exchange of calque and loanwords between languages, and to their importation from other languages, there are few concepts that are "untranslatable" among the modern European languages.

A greater problem, however, is translating terms relating to cultural concepts that have no equivalent in the target language. For full comprehension, such situations require the provision of a gloss. For the most part, the more prominent the contact and trade that have existed between two dialects, or between those dialects and a third one, the more noteworthy is the proportion of metaphrase to reword that might be utilized in deciphering among them. Propelled in April 2006 as a factual machine interpretation administration, it utilized United Nations and European Parliament transcripts to accumulate semantic information. As opposed to interpreting dialects legitimately, it initially makes an interpretation of content to English and afterward turns to the objective language in a large portion of the language blends it places in its network, with a couple of special cases including Catalan-Spanish.

During an interpretation, it searches for designs in a large number of reports to help settle on which words to pick and how to organize them in the objective language. Google has publicly supporting highlights for volunteers to be a piece of its "Make an interpretation of Community", proposed to help improve Google Translate's precision. Because of contrasts between dialects in speculation, look into, and the degree of computerized assets, the exactness of Google Translate changes enormously among dialects. A few dialects produce preferred outcomes over others. Most dialects from Africa, Asia, and the Pacific, will in general score ineffectively according to the scores of some very much financed European dialects, with Afrikaans and Chinese being the high-scoring special cases from their mainland. Since Google Translate utilized factual coordinating to decipher, interpreted content can frequently incorporate evidently silly and clear errors,sometimes swapping normal terms for comparative however nonequivalent basic terms in the other language or modifying sentence meaning.

\section{RELATED WORKS}

There are numerous potential applications for polylingual subject models. Despite the fact that exploration writing is normally written in English, bibliographic databases frequently contain generous amounts of work in different dialects. To perform point put together bibliometric investigation with respect to these assortments, it is important to have theme models that are adjusted across dialects. Bilingual point models for equal writings with word-to-word arrangements have been examined already utilizing the HMbitam model. In any case, they assess their model on just two 
dialects (English and Chinese), and don't utilize the model to recognize contrasts between dialects. practically identical writings may not utilize the very same points, it turns out to be urgently imperative to have the option to describe contrasts into picprevalence at the archive level and at the languagewide level. Both of these interpretation centered point models derive word-to-word arrangements as a component of their induction systems, which would turn out to be exponentially increasingly intricate if extra dialects were included. Easier methodology that is increasingly appropriate for topically comparable archive tuples (where reports are not immediate interpretations of each other) in multiple dialects.

Coordinating is helpful in different applications including a scope of estimation sources that are not legitimately commensurable. Expecting shared data as the proportion of reliance, the issue is to find the coordinating that boosts it. practically speaking, estimating shared data between two discretionary vectoral estimations is difficult and thus approximations are required. Straightforwardly expanding Hilbert-Schmidt Independence Criterion (HSIC) brings about a mind boggling calculation that requires approximative arrangements. Enormous sentence-adjusted corpora are required for learning measurable machine interpretation models. The sentence arrangement is ordinarily founded on accessible "stay" signals, (for example, speaker identifiers and section markers) and sentence lengths. Coordinating examples of two un-requested information lattices is a general issue with applications in a scope of areas. Not at all like run of the mill sentence arrangement strategies utilized practically speaking, we don't utilize data like stay signs, sentence length, or interpretation vocabularies. Such wellsprings of data could anyway be abused, for instance, as earlier information to find halfway arrangements, or for acquiring optional portrayals that would be straightforwardly commensurable.

Most article coordinating techniques require comparability quantifies between objects in various spaces, or correspondence information for learning the closeness measures. These strategies find just balanced matching between objects. Be that as it may, in certain applications it is proper to find many-to-many coordinating. Rematch expect that the given various systems have regular idle gatherings, where each gathering displays a specific cooperation design with different gatherings. Rematch doesn't expect that correspondence data between hubs in various systems is given ahead of time or even conceivable to acquire. Client identifiers probably won't be shared between various organizations, arrangements probably won't be accessible in minor dialects, and morphological likeness can't be accepted between dialects utilizing various characters. The strategy accept Gaussian clamor for input information, it isn't appropriate for arrange or social information. Discovering correspondence between numerous systems is identified with arrange de-anonymisation given different systems.

\section{EXISTING SYSTEM}

Documents can be translated into only two languages. Those are chinese and german. While translating documents from one language to another user has to use another application. Encryptions have lots problems, it has only 64 bit encryption, which leaves easy way to attackers to steal user files. In existing system while translating document from one language to another meaning of the documents are changing.

\section{PROPOSED SYSTEM}

Proposing a method which can translate document into user define language. With this user need not to bother for translating to own language according to user wish. User can easily translate their document while downloading file from server itself only. Server can store any documents in it. While storing the documents in server, used RSA256 bit encryption. This provides more security than existing system. Because existing has only 64bit encryption which is easy to break security levels. RSA256 has more rounds of encryption so its very hard to break the security levels of documents. User can translate their document into 56 languages. But this process required constant internet connection because translating while downloading itself need internet. When user translates documents, translator won't change meaning of document. Translator give correct meaning of document and user can easily understands meaning or syntax of documents. Whenever server gets huge requests from internet or users then it gives error message because of security reasons. Server thinks that as DOS attack. To avoid user collisions it shows error message to users.

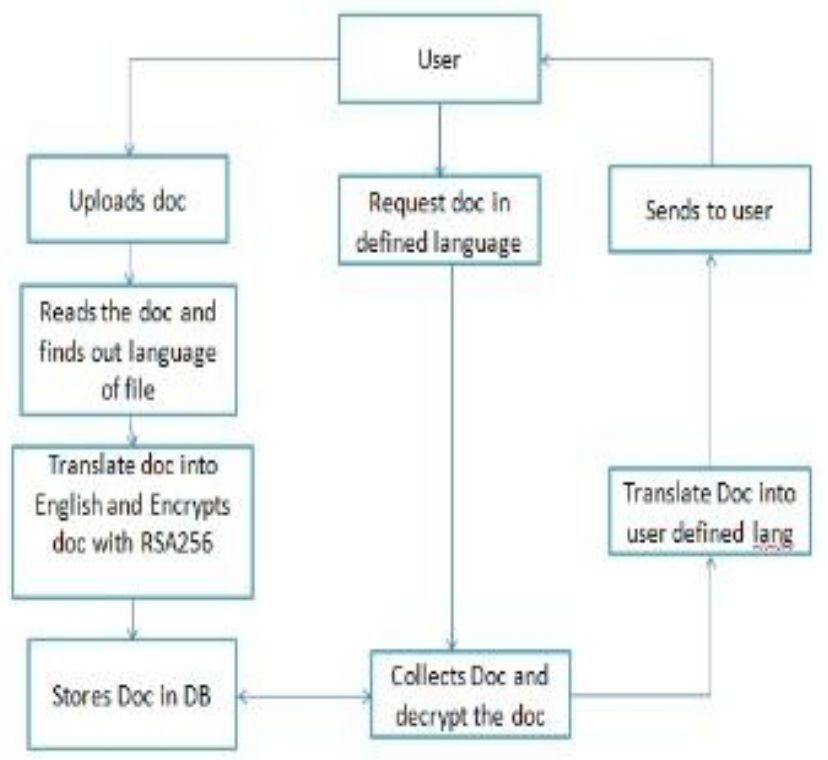

Figno-1Architecture model

\section{CONCLUSION}

With the proposing method user can easily translate any document into any language according to user wish which is supported by translator. RSA256 bit encryption applied for user documents in the server level. It gives better security to server as well as user documents or files. It consumes less amount of resources and works efficiently. Users can translate document while downloading itself only. No need to go for any other applications for translating the documents.

\section{REFERENCES}

[1] "PolylingualTopicModels" Proceedings of the 2009 Conference on Empirical Methods in Natural Language Processing, pages 880-889, Singapore, 6-7 August 2009.

[2] "BILINGUAL SENTENCE MATCHING USING KERNEL CCA" 2010 IEEE International Workshop on Machine Learning for Signal Processing (MLSP 2010) August 29 - September 1, 2010, Kittilä, Finland.

[3] "Unsupervised Many-to-Many Object Matching for Relational Data" IEEE TRANSACTIONS ON PATTERN ANALYSIS AND MACHINE 


\section{INTELLIGENCE.}

[4] "Document Recognition/Aut henticat ion Based on Medium-Embedded Random Patterns" https://ieeexplore.ieee.org/document/395774

[5] "ONLINE BINARY VISUALIZATION FOR PDF DOCUMENTS" 978-1-5386-4615-1/18/\$31.00 (O2018 IEEE

[6] "Scalability Analysis of Semantics based Distributed Document Clustering Algorithms "2017 International Conference on Intelligent Computing,Instrumentation and Control Technologies (ICICICT) https://ieeexplore.ieee.org/document/8342660

[7] "An improved Document Clustering Approach with Multi-Viewpoint based on different similarity measures "Proceedings of the Second International Conference on Intelligent Computing and Control Systems (ICICCS 2018) IEEE Xplore Compliant Part Number: CFP18K74-

\section{ART; ISBN:978-1-5386-2842-3.}

[8] "Efficient Phrase-Based Document Indexing for Web Document Clustering" IEEE TRANSACTIONS ON KNOWLEDGE AND DATA ENGINEERING, VOL. 16, NO. 10, OCTOBER 2004.

[9] "Document Clustering Method using Weighted Semantic Features and Cluster Similarity" 2010 IEEE International Conference on Digital Game and Intelligent Toy Enhanced Learning.

[10] "Clustering Web Retrieval Results Accompanied by Removing Duplicate Documents" 2010 International Conference on Web Information Systems and Mining.

[11] N. Quadrianto, A. J. Smola, L. Song, and T. Tuytelaars, "Kernelized sorting," IEEE Trans. on Pattern Analysis and Machine Intelligence,zvol. 32, no. 10, pp. 18091821, 2010. 